\title{
Atenção Primária em Saúde no cuidado ao idoso dependente e ao seu cuidador
}

\author{
Primary Health Care in caring for dependent older adults \\ and their caregivers
}

Roger Flores Ceccon (https://orcid.org/0000-0002-0846-1376) ${ }^{1}$

Konrad Gutterres Soares (https://orcid.org/0000-0002-5361-9419) ${ }^{2}$

Luiza Jane Eyre de Souza Vieira (https://orcid.org/0000-0002-5220-027X) ${ }^{3}$

Carlos Alberto Severo Garcia Júnior (https://orcid.org/0000-0003-3367-4151) ${ }^{1}$

Camila Carvalho de Souza Amorim Matos (https://orcid.org/0000-0003-0295-4230) ${ }^{3}$

Macelle Dias de Holanda Alencar Pascoal (https://orcid.org/0000-0002-9707-8618) ${ }^{3}$

${ }^{1}$ Departamento de Ciências da Saúde, Universidade Federal de Santa Catarina. R. Gov. Jorge Lacerda 3201, Urussanguinha. 90620-110 Araranguá SC Brasil.

roger.ceccon@hotmail.com ${ }^{2}$ Faculdade de Medicina,

Universidade Federal do Rio

Grande do Sul. Porto Alegre

RS Brasil.

${ }^{3}$ Programa de Pós-

Graduação em Saúde

Coletiva, Universidade de

Fortaleza. Fortaleza, CE.

\begin{abstract}
Primary Health Care is a care model whose attributes contribute to solving most of the health problems of older adults in Brazil's increased longevity. This investigation aims to analyze the care provided to the dependent older adults and their caregivers within Primary Health Care. This is a qualitative study conducted in eight Brazilian municipalities in 2019. A total of 190 subjects participated in the research, whose information was collected through semi-structured interviews and analyzed through the theoretical framework of Dialectic Hermeneutics. Problems with access, home care, the health care network, and interprofessional work were identified. The teams provide practices under the biomedical model's logic focused on the medical professional, although health promotion and disease prevention actions have been identified. There is a need to qualify PHC and expand the scope of practices, incorporating the core of knowledge not traditionally introduced into the teams. Moreover, it is essential to strengthen the State's role and create specific public policies for dependent older adults and their caregivers.
\end{abstract}

Key words Primary Health Care, Elderly, Dependence, Caregivers, Qualitative research
Resumo A Atenção Primária em Saúde é um modelo de cuidado cujos atributos contribuem para resolver a maioria dos problemas de saúde dos idosos, em um contexto de aumento da longevidade no Brasil. Esta investigação tem como objetivo analisar o cuidado dispensado ao idoso dependente e seus cuidadores no âmbito da Atenção Primária à Saúde. Trata-se de um estudo qualitativo realizado em oito municípios brasileiros no ano de 2019. Participaram da pesquisa 190 sujeitos, cujas informações foram coletadas por meio de entrevistas semiestruturadas e analisadas através do marco teórico da Hermenêutica Dialética. Foram identificados problemas no acesso, na atenção domiciliar, na rede de atenção à saúde e no trabatho interprofissional. As equipes ofertam práticas sob a lógica do modelo biomédico e centradas no profissional médico, embora tenham sido identificadas ações de promoção de saúde e prevenção de doenças. Há necessidade de qualificar a APS e ampliar o escopo de práticas, incorporando núcleos de saberes que não estão tradicionalmente inseridos nas equipes. Além do mais, é fundamental o fortalecimento do papel do Estado e a criação de políticas públicas específicas para os idosos dependentes e seus cuidadores.

Palavras-chave Atenção Primária em Saúde, Idoso, Dependência, Cuidadores, Pesquisa qualitativa 


\section{Introdução}

O Brasil ultrapassou a marca de 30 milhões de idosos e atingiu 14\% da população total, acendendo em pessoas de 80 anos ou mais. Assim, a manutenção da autonomia e da independência tornou-se um desafio para este grupo, pois são suscetíveis a doenças e agravos incapacitantes, necessitando do auxílio de cuidadores por longos períodos ${ }^{1}$.

$\mathrm{O}$ "cuidado de longa duração" à pessoa idosa compõe práticas assistenciais não especializadas direcionadas às atividades da vida diária (AVD) $\mathrm{e}$ às atividades instrumentais da vida diária (AIVD). Estes podem ser ofertados na comunidade, no domicílio ou em instituições, embora os serviços disponíveis se mostrem inadequados e/ ou insuficientes. Para viabilizar essa assistência, a responsabilidade recai sobre familiares ou pessoas contratadas, muitas vezes também idosas e com agravos de saúde ${ }^{1,2}$.

Os direitos assegurados aos idosos ${ }^{3}$, incluindo à saúde, não estão integralizados ao cotidiano dessa população. Estudos apontam a importância de os serviços de saúde fomentarem ações preventivas das condições crônicas, com cuidado interdisciplinar, priorizando o modelo socioambiental de atenção à saúde, considerando os idosos no seu contexto de vida e privilegiando cuidados primários em saúde ${ }^{1,4}$.

Conjugada às recomendações desses estudos, a Atenção Primária à Saúde (APS) é um modelo de atenção cujos atributos cooperam para resolver a maioria dos problemas de saúde da população idosa, reduzindo intervenções desnecessárias, ampliando o acesso aos serviços e favorecendo a atenção integral aos diferentes problemas ${ }^{5,6}$. A Estratégia de Saúde da Família (ESF), modelo brasileiro de efetivação da APS, fundamenta-se na atenção à saúde ao idoso e seu cuidador, incluindo ações individuais e coletivas de promoção à saúde, prevenção de doenças, diagnóstico, tratamento, reabilitação, redução de danos e cuidados paliativos ${ }^{7}$.

No entanto, o cuidado integral dispensado à díade idoso-cuidador enfrenta percalços que demanda articulação política, ressignificação sociocultural, reestruturação dos serviços de saúde e dos equipamentos sociais, remodelação das práticas de cuidado e olhar plural à atenção domiciliar. E nessa elaboração lógica, o cuidado ofertado pela APS ao idoso e ao seu cuidador exige ações e serviços que abranjam as especificidades destes usuários e familiares, em busca de práticas efetivas e orientadas pelas políticas públicas existentes.
Portanto, indaga-se como a APS se organiza na atenção ao idoso dependente e seus cuidadores, bem como é reconhecida diante da heterogeneidade organizacional e assistencial no Brasil. Nessa ótica, o estudo analisa o cuidado dispensado ao idoso dependente e seus cuidadores no âmbito da APS.

\section{Método}

Estudo qualitativo cujo marco teórico se insere na perspectiva da hermenêutica-dialética ${ }^{8}$, o qual valorizou o exercício crítico e compreensivo da linguagem, das relações e das práticas sociais das pessoas envolvidas com a dependência dos idosos no Brasil. Faz parte de pesquisa multicêntrica cujo foco é formular subsídios para a construção de políticas públicas sobre dependência ${ }^{9}$, ponderando o cenário de crescimento da longevidade ${ }^{10}$ em um país inscrito nas desigualdades sociais ${ }^{11}$.

A investigação, durante o ano de 2019, abrangeu oito municípios brasileiros: Araranguá (SC), Brasília (DF), Fortaleza (CE), Manaus (AM), Porto Alegre (RS), Recife (PE), Rio de Janeiro $(\mathrm{RJ})$ e Teresina (PI). Estes representam regiões do país que apresentaram envelhecimento populacional nos últimos anos e heterogeneidades sociodemográficas e culturais ${ }^{11}$.

Participaram da pesquisa 190 sujeitos; 64 idosos com dependência; 27 cuidadores formais; 72 cuidadores familiares; 7 gestores; e 20 profissionais que atuam na APS. Primeiramente, os idosos e os cuidadores foram identificados por meio da Secretaria Municipal de Saúde e dos serviços de APS de cada município. Após, procedeu-se o contato e o agendamento das entrevistas, sendo realizadas nas residências dos idosos e cuidadores. Os gestores e os profissionais foram entrevistados nos respectivos locais de trabalho.

Incluíram-se pessoas idosas com dependência física, mental ou cognitiva, de ambos os sexos, aptos a responderem a entrevista; cuidadores domiciliares formais ou familiares, maiores de 18 anos; profissionais de saúde que atuam na APS; e gestores (secretários de saúde, gestores da APS ou específicos de programas de idosos). Excluíramse os idosos vinculados à Instituições de Longa Permanência ou residindo sozinhos. Considerou-se dependência física a incapacidade funcional, prática ou motora para realizar ABVD ou AIVD; e dependência cognitiva e mental a perda completa ou parcial da orientação no tempo, da memória, atenção, realização de cálculo, linguagem e capacidade visual ${ }^{12}$. 
Entrevistas semiestruturadas, orientadas por um instrumento construído por pesquisadores de diferentes universidades brasileiras, com questões específicas para cada grupo entrevistado (idosos, cuidadores, gestores e profissionais) nortearam a coleta das informações. Esta versou sobre as características sociodemográficas, as situações de vida, as vivências e a dependência funcional, cognitiva, mental, emocional e social do idoso; percepção dos cuidadores, profissionais e dos gestores sobre os idosos, incluindo facilidades, dificuldades e iniciativas de assistência à saúde.

A técnica de Análise de Conteúdo do tipo Temática orientou o tratamento das informações em três etapas: (1) pré-análise, com a realização das sínteses analíticas de cada entrevista transcrita, compilação e organização em um corpus textual, acrescida de leitura flutuante; (2) exploração do material, com a elaboração das categorias analíticas oriundas do texto, principalmente relativas aos aspectos que interligam a dependência com a gestão e assistência ofertada na APS; (3) tratamento das informações e interpretação, com inferências procedentes da compreensão e do exercício crítico das falas dos participantes, valorando os significados das narrativas dos sujeitos ${ }^{8}$.

Alinhado ao objetivo do estudo e às falas dos participantes, construíram-se categorias temáticas relativas à gestão e assistência em ações ao cuidado do idoso dependente: (1) Trabalho em equipe e Práticas de cuidado; (2) Acesso à APS e Atenção Domiciliar. Embora o cenário da pesquisa se constitua por diferentes localidades brasileiras, não foi objeto deste estudo analisar as especificidades loco-regionais, mas as situações comuns relatadas pelos entrevistados, especialmente no que se refere à micropolítica e ao cotidiano de trabalho da APS no cuidado ao idoso dependente.

O projeto foi aprovado pelo Comitê de Ética e Pesquisa da Fundação Oswaldo Cruz.

\section{Resultados e discussão}

A APS é um modelo assistencial na garantia do acesso aos idosos dependentes e seus cuidadores a práticas integrais de saúde, sob a lógica de trabalho em equipe com diferentes núcleos de saberes e práticas, especialmente no cuidado domiciliar ${ }^{5,6}$. Privilegiou-se neste estudo a análise de situações que reafirmam a importância da APS na garantia deste escopo de práticas e aspectos que sinalizam fragilidades, apresentando subsí- dios à qualificação e à garantia da integralidade da atenção em saúde ao idoso dependente.

Com relação às características dos entrevistados, a maioria dos idosos era mulher, com idade superior a 80 anos, da raça/cor branca, com baixa escolaridade e de religião católica; os cuidadores familiares e formais eram, em sua maioria, do sexo feminino e tinha entre 40 e 59 anos, diferindo-se na característica relativa à raça, já que a maioria dos cuidadores formais era negra e os familiares de cor da pele branca. Dentre os profissionais de saúde e gestores, a maioria era do sexo feminino, enfermeira e com idade entre 30 e 50 anos.

\section{Trabalho em equipe e práticas ofertadas na APS aos idosos dependentes}

A categoria analisa como os diferentes núcleos de profissionais da saúde são reconhecidos quanto à produção do cuidado, assumindo $N u$ cleo como a identidade de uma área a partir da concentração de saberes e práticas profissionais; e campo o espaço de limites imprecisos onde cada disciplina e profissão busca em outras o apoio para cumprir seus objetivos ${ }^{13}$.

As falas dos participantes concentram-se (1) no cuidado centralizado no profissional médico, com práticas restritas ao processo saúde-doença e na medicalização; (2) na importância dos Agentes Comunitários de Saúde (ACS), com práticas individuais e coletivas à promoção de saúde e prevenção de doenças; e (3) nos arranjos organizacionais que ampliam o escopo de práticas, incluindo profissionais de diferentes núcleos de saberes.

Considerando que a APS preconiza o trabalho em equipe, com relações democráticas de saber e poder, observou-se aspectos que fragilizam o exercício da interprofissionalidade no cuidado ao idoso dependente. Os relatos indicam que os profissionais da equipe não participam do cuidado na mesma intensidade, nem ofertam práticas integradas e colaborativas:

Vou no postinho e o médico vem fazer a visita domiciliar. Muito bom, muito atencioso, muito querido. Tudo que eu preciso eu tenho. Vou ali e ele me consegue (Cuidadora familiar, 62 anos, Araranguá).

A enfermeira vinha fazer a vacina e curativo, agora não tá vindo mais. Mas o médico que me acompanha é do plano de saúde (Idosa, 78 anos, Rio de Janeiro).

Embora muitas das necessidades dos idosos pudessem ser sanadas por cuidados interdisci- 
plinares, os relatos reiteram a tênue vinculação de ações aos determinantes sociais em saúde, evidenciando a dificuldade de o profissional transpor as raízes do cuidado biomédico para o integral. Essas situações retratam a perspectiva hospitalar, especializada e circunscrita na doença, paradigmas históricos e hegemônicos no Brasil. Os usuários não reconhecem a APS como um dispositivo capaz de ofertar práticas que compreendam saúde em uma perspectiva ampliada, que envolve aspectos sociais, econômicos, políticos, culturais e mentais, restringindo o cuidado ao corpo biológico do idoso ${ }^{14}$.

A maioria dos entrevistados se refere à Unidade de Saúde como seu "postinho" e não reconhece o conjunto de profissionais da equipe, referem-se ao médico como o responsável por diagnosticar e tratar doenças. A equipe de enfermagem é vista como procedimental, responsável por aplicação de vacina, sondagem e curativos, e a saúde bucal não aparece nos relatos.

$\mathrm{O}$ trabalho em equipe mantém-se em geral fragmentado, hierarquizado e assimétrico, com subordinação de diversos núcleos às práticas médicas. Essas distorções fragilizam o trabalho interprofissional, cujo princípio é a valorização de diferentes saberes para assegurar atenção qualificada às necessidades dos idosos ${ }^{15}$.

O saber biomédico influi nas práticas de saúde e na subjetividade das pessoas em relação ao corpo e à vida ${ }^{16}$ e mostrou-se cíclico com a recorrência de expressões conexas a medicamentos, exames, cirurgias, vacinas e procedimentos. A visão crítica do enfermeiro e o relato da idosa provam a hegemonia desse saber:

A gente acaba criando uma medicalização, a gente medica, medica, dá, dá, dá remédio, mas ao mesmo tempo não sabe qual o contexto social dele, dá trezentos remédios e não sabe se ele lê! (Enfermeiro, 40 anos, Porto Alegre).

Quando o médico pede exame, pede logo e muito, seis, sete exames. Eles vêm aqui e recolhem meu sangue (Idosa, 83 anos, Manaus).

A medicação é a prática mais citada como cuidado na APS, ainda que busque atuar sobre as condições e os contextos socioeconômicos e a história de vida do idoso e seus familiares. Entretanto, essas práticas carecem vincular-se às ações compartilhadas pela equipe de saúde, pertinentes à realidade e necessidade dos territórios e dos indivíduos ${ }^{17}$.

Os idosos dependentes e seus cuidadores, por vezes, anseiam respostas focadas na doença, na biomedicina e nas tecnologias duras, em detrimento das tecnologias leves que priorizam os saberes relacionais ${ }^{18}$. A apreciação apenas dos saberes estruturados e recursos materiais estabelece uma "cultura biomédica" de cuidado, reflexo da compreensão da saúde sob a égide do mercado farmacológico e do discurso dos riscos à saúde ${ }^{19}$.

É basilar a constituição de um trabalho relacional, interprofissional e corresponsável pelo cuidado em equipe ${ }^{20}$. Ainda é imperioso um olhar atento às necessidades de saúde dos idosos, envolvendo aspectos que transcendem condições biológicas e práticas médicas, conforme diz o idoso: Tô acabado agora, desesperado, eu choro sozinho. Não posso fazer nada, ganho só um salário mínimo (Idoso, 89 anos, Araranguá).

Embora a racionalidade hegemônica volte-se ao paradigma biomédico, evidenciou-se estratégias de organização do processo de trabalho na APS para a constituição de práticas de apoio ao idoso e seu cuidador com tecnologias leves. Essas práticas de cuidado acabam formando a caixa de ferramentas para outras apostas na produção de saúde, valorizando atividades coletivas, integrativas e complementares. No trecho tem-se exemplo destas apostas de cuidado:

A gente se organiza para acolher esses idosos em grupos. O grupo da terceira idade é responsabilidade da fisioterapia. A Oficina da Memória com a terapeuta ocupacional. Temos parceria com o CRAS onde a assistente social faz oficina de trabalhos manuais, o grupo das "Empoderadas" ('Gestora, 54 anos, Rio de Janeiro).

Os grupos são dispositivos fundamentais para a constituição de vínculos e socialização para idosos e familiares na APS. Acompanhados de modo interprofissional, tornam-se resolutivos, incorporam e ampliam práticas relacionadas às demandas dos idosos dependentes e seus cuidadore $^{21}$. Nesse sentido, o ACS é reconhecido pelos entrevistados pelo conjunto de saberes e práticas que se aproxima com a lógica de produção de saúde que não está centrada na doença. Constitui um núcleo fundamental na assistência ao idoso, elo entre a equipe e o contexto familiar, alguém que conhece as dinâmicas culturais do território e oferta práticas coletivas de promoção de saúde:

O agente de saúde é fundamental porque está no território, sabe onde estão os idosos. A gente cadastra, faz estratificação e ele sabe quem são essas pessoas, sabe a realidade da família e onde estão. Eles também fazem grupos cooperativos (Gestor, 42 anos, Fortaleza).

O papel do ACS no cuidado ao idoso dependente é de fundamental importância, pois o conjunto de incapacidades limita esse usuário a 
procurar o serviço de saúde, gerando demanda de visitas domiciliares periódicas. É importante que os ACS realizem busca ativa, identifiquem vulnerabilidades, efetivem atividades no domicílio e comunidade ${ }^{22}$ e tornem a visita domiciliar uma ferramenta de cuidado que contempla os aspectos sociais, econômicos, relacionais e psicológicos, pois seu escopo de práticas acontece no contexto onde vivem os idosos ${ }^{23}$.

Embora estudos atestem o valor do trabalho do $\mathrm{ACS}^{20,22}$ e os entrevistados desta pesquisa o reconheça, a Política Nacional de Atenção Básica, reformulada em 2017, fragiliza a atuação deste profissional ao reduzir o número mínimo de ACS por equipe. Recomendar o acompanhamento de usuários mais vulneráveis (sem parâmetros objetivos), e não mais a população do território ${ }^{24}$, compromete a integralidade do cuidado, especialmente, aos idosos dependentes que podem não ser compreendidos como "mais vulneráveis" ou que sequer sejam identificados.

Observou-se neste estudo que os idosos dependentes enfrentam desafios que vão além das doenças, compreendendo as incapacidades funcionais, violências, negligência, pobreza, alimentação inadequada, problemas emocionais e familiares e ausência de redes sociais de apoio. Além do mais, o trabalho dos cuidadores é permeado por dúvidas, incertezas e precarizações. Isso impõe a necessidade de inserir profissionais que não estão tradicionalmente previstos nas equipes da APS, reduzindo os encaminhamentos dos idosos para outros pontos da Rede de Atenção à Saúde, já que pode gerar demora e deslocamento de usuários que não possuem condições de locomoção. Esse fato extrapola saberes e práticas oriundos da medicina-enfermagem, tradicionalmente inseridas na APS, e coaduna conhecimentos de outros núcleos de saberes:

Gostaria que o psicólogo visitasse nossa casa. Esse profissional é ótimo. Tem hora que o idoso tá enjoado das nossas conversas. Só a nora e os netos. Fisioterapeuta também seria muito bom (Cuidadora familiar, 38 anos, Manaus).

Tem Unidades que tem o NASF, tem assistente social e ajuda muito. Tem o grupo da terceira idade, o chefe é da fisioterapia. Tem a Oficina da Memória que é com o terapeuta ocupacional (Gestora, 54 anos, Rio de Janeiro).

Para o atendimento integral e resolutivo dessas necessidades é mandatório o cuidado produzido por "especialistas", cujo núcleo de saber e prática não está inserido nas equipes da APS. Embora muitas dessas situações possam ser abordadas a partir da noção de campo que compõe as práticas profissionais, as condições dos idosos exigem cuidados específicos, como o psicólogo nos casos de sofrimento mental, o fisioterapeuta e o terapeuta ocupacional nos eventos de reabilitação e o nutricionista na condução da segurança alimentar.

Entre os problemas que contribuem para a baixa resolutividade da APS está a responsabilização clínica insuficiente e inadequada, decorrentes da racionalidade hegemônica, que reduz o objeto de trabalho a procedimentos, doenças ou partes do corpo, em detrimento de um cuidado centrado na pessoa. Esta inversão requer profissionais de diferentes áreas, pois seus escopos de práticas são limitados e focais ${ }^{13}$.

Quando necessário, ocorrem encaminhamentos desses idosos para especialistas, em diferentes serviços e ausência de vínculo, além das filas de espera. Tem-se um tipo de racionalidade burocrática que pode exacerbar a fragmentação da atenção, ao desvalorizar o vínculo equipe-usuário $^{23}$. Em muitas situações, encaminha-se para locais longínquos de onde residem, dificultando não só o acesso, mas a integração entre os serviços de saúde:

A doutora me passou para o cardiologista $e$ para o ortopedista. Ainda não consegui a consulta para nenhum dos dois. Só dependo do cardiologista para ver se sou hipertensa (Cuidadora familiar, 62 anos, Manaus).

$O$ relato da cuidadora, que também refere adoecimento, mostra o quanto os modelos de atenção à saúde apresentam fragmentação da clínica, tanto no próprio serviço quanto externamente, valorizando de forma desigual os diferentes saberes. Para superar estes desafios, um dos arranjos citados é a institucionalização do apoio matricial por meio do Núcleo Ampliado de Saúde da Família (NASF), buscando assegurar retaguarda especializada a equipes e profissionais da APS. Trata-se de uma metodologia de trabalho complementar à prevista em sistemas hierarquizados, que se utilizam de mecanismos de referência e contrarreferência, protocolos e centrais de regulação ${ }^{25}$.

Embora estudos evidenciem a importância do NASF, no ano de 2019, pela Portaria 2.979, foi extinto como política de Estado com financiamento do governo federal ${ }^{26}$. Entretanto, os profissionais não foram incluídos nas equipes de Saúde da Família, o que possivelmente dificultará o acesso a especialistas e contribuirá para agravar o cuidado ao idoso dependente. Representa a redução do papel do Estado e limita a garantia de direitos a populações em situação de vulnerabilidades. 
Por fim, os achados evidenciam que a complexidade das condições em que vivem os idosos dependentes exige a necessidade de considerar novos arranjos nas equipes de saúde, com a inserção de outros núcleos profissionais, cujas práticas extrapolem a doença e o modelo biomédico. Assegurar a garantia da integralidade do cuidado ao idoso é imperativo, além do trabalho interprofissional, em equipe, o fortalecimento da atuação dos ACS e o combate à precarização do trabalho na APS.

\section{Acesso e cuidado domiciliar na APS: integralidade e proteção social ao idoso dependente}

Esta seção apresenta os achados relativos ao acesso dos idosos dependentes à APS e as estratégias para a produção do cuidado por meio da atenção domiciliar, reiterando que um dos atributos essenciais da APS é o primeiro contato, constituindo-se porta de entrada para o sistema de saúde ${ }^{9}$. Nesse sentido, dificuldades são registradas pelos entrevistados:

As pessoas chegam na unidade meia-noite, uma, duas horas da madrugada. Antes, quando eu ia, o meu sobrinho pagava. Sempre tem aquelas pessoas que ficam na fila, dá vinte reais. Meu sobrinho me leva para lá 7h30, fico até a hora de abrir. Disseram que eles vão em casa, estou esperando até hoje (Idosa, 89 anos, Porto Alegre).

Os desafios apontados pelos idosos e cuidadores para acessar o serviço de saúde da APS foram inúmeros, desde barreiras geográficas até à organização do processo de trabalho da equipe de saúde. Em se tratando de idosos dependentes, essas questões apresentam-se mais complexas, pois muitas vezes os idosos têm mobilidade reduzida, dependem do outro para se deslocar ou seu acesso necessita de reorganização do processo de trabalho, com preferência para visitas domiciliares ${ }^{27}$.

O cuidado domiciliar, principalmente dos que não podem se deslocar até o serviço de saúde, é uma das prioridades da $\mathrm{APS}^{28}$. A atenção domiciliar $(\mathrm{AD})$ às pessoas idosas envolve ações realizadas pela equipe no domicílio, favorecendo o desenvolvimento e a adaptação de suas funções de maneira a restabelecer a independência e autonomia ${ }^{29}$.

A AD é um dos pontos de atenção à saúde que deve ser realizada por profissionais das equipes e contribuir no acesso dos idosos dependentes às ações de saúde. A gestão do cuidado domiciliar pode ser compreendida nas dimensões profissio- nal, caracterizada pelo momento do encontro entre trabalhador e usuário; organizacional, que diz respeito à institucionalização das práticas, como a organização do processo de trabalho e registro das informações; e sistêmica, considerando os serviços de saúde, seus diferentes papeis e a incorporação tecnológica para garantir a integralidade na assistência. A interdependência das três dimensões é fundamental para os serviços de atenção domiciliar ao idoso dependente $e^{20,30}$.

Estudo realizado no município de Porto Alegre mostra que menos da metade dos idosos referiu acessar habitualmente o serviço da APS, apontando que metade dos serviços correspondia ao preconizado quanto ao atributo de primeiro contato. Sendo as unidades da APS porta de entrada do sistema de saúde, o nível de facilidade ou dificuldade encontrado para acessá-las determina o percurso que esse indivíduo irá traçar na rede de atenção em saúde na busca por cuidado ${ }^{31}$. Eis a estratégia para facilitar o acesso dos idosos:

No Posto onde trabalho, tem reserva especifica de vagas do idoso feita por telefone. É uma estratégia que a gerente negociou para evitar que ele tenha que ir até o Posto e ficar em fila ou esperando no guichê. Então ele liga para lá e é marcada a consulta (Enfermeiro, 40 anos, Porto Alegre).

Para suprir a necessidade dos idosos dependentes, o cuidado exige diferentes arranjos na organização do trabalho e, por mais que ações institucionais à nível de gestão municipal sejam importantes, cabe às equipes avaliar a melhor estratégia para atender as demandas da população adstrita. A reserva de vagas, a atenção domiciliar, o acolhimento, o acesso avançado e a lista de pacientes são mecanismos que podem superar as barreiras do acesso.

Não apenas os idosos e seus cuidadores referiram dificuldades no acesso e no cuidado domiciliar, mas também os profissionais de saúde e gestores, com ênfase na insuficiência de transporte para visitas domiciliares, a adscrição populacional maior do que o preconizado e a alta demanda por atendimentos clínicos. No âmbito organizacional e alusivo à integralidade, a organização da agenda de visitas e a disponibilidade de transporte são obstáculos perenes:

Um idoso dependente, se for totalmente restrito ao lar e precisa de visita domiciliar, nós temos um dia para quatro equipes de saúde da família. Isso restringe o acesso, porque acaba tendo equipes que só fazem visitas de quinze em quinze dias, por conta da disponibilidade do carro, e as famílias não têm condições de pagar para trazer o idoso (gestor, 46 anos, Fortaleza). 
As condições para um processo de trabalho qualificado e um planejamento prévio às visitas domiciliares são estratégias para a resolutividade da AD. Contudo, a solução para as barreiras do acesso ao cuidado domiciliar também está condicionada às diferentes instâncias gestoras. Enquanto um está sujeito à organização dos profissionais das equipes, o outro depende da condução da gestão municipal. Portanto, a dimensão do cuidado sistêmico e socioambiental é necessária para reforçar os acordos da APS com os idosos dependentes e seus familiares.

Com relação a essas dificuldades e facilidades observadas pelos profissionais de saúde no atendimento às pessoas idosas, um debate vem à tona: ações programáticas e agendas fechadas versus demanda espontânea:

Não existe facilidade para o idoso, existe um sistema de marcação rigoroso. No dia da demanda tem que ver se tem vaga. $O$ idoso tem mais comorbidades. Ele ou algum parente tem que vir e tentar agendar consulta. Depende da disponibilidade da agenda (Médico, 44 anos, Fortaleza).

Às vezes quando eles não conseguem vaga na agenda, você consegue atender na demanda espontânea com o acolhimento, que a maioria das vezes é só renovação de receita (Enfermeira, 41 anos, Fortaleza).

As questões da agenda perpassaram as falas, na maioria das vezes para justificar as dificuldades de acesso dos idosos às consultas. A demanda espontânea, por sua vez, içou discordâncias: alguns identificam como potencialidade, e outros que as atividades de educação e promoção em saúde estão perdendo espaço com a ampliação desse tipo de demanda.

O Ministério da Saúde orienta que as equipes organizem o acesso dos idosos por meio do acolhimento, compreendendo que os indivíduos também são capazes de definir o que é saúde e o que é necessidade; que boa parte das demandas podem ser resolvidas na APS; que a demanda espontânea pode ser aproveitada para estabelecimento de vínculo; e que, mesmo os idosos acompanhados longitudinalmente em ações programáticas, podem apresentar quadros diferentes daqueles pelos quais são acompanhados, necessitando de atendimentos em momentos não programados. Neste último grupo encontram-se muitos dos idosos dependentes ouvidos nesta pesquisa, como pode ser visto nos excertos aqui discutidos ${ }^{27}$.

$\mathrm{O}$ acesso ao cuidado domiciliar é peça fundamental para estabelecer relação entre equipe e família e extrapolar a noção de prevenção de doenças e incorporar propostas de promoção de saúde. Mazza e Lefèvre ${ }^{32}$ asseveram dois tipos de cuidado domiciliar: técnico, ofertado pelos profissionais de saúde, e leigo, resultado da intuição e suporte nas AVDs ao idoso. Assim, pode-se considerar, em alguns casos, a existência de uma "disputa" de planos de cuidado, na qual o cuidador assume parte dos cuidados e a equipe de saúde oferta outros ${ }^{33}$.

Um dos principais aspectos da vida da pessoa idosa é a relação que estabelece com seu cuidador, no qual também surgiu nos relatos sobre o acesso à APS:

Não existe política do SUS para cuidadores. Dificilmente a gente pensa no cuidador. Muitos não conseguem sair de casa pois se dedicam a essas pessoas. A pessoa muda a vida dela totalmente para cuidar daquele idoso. Então, para vir na unidade, precisaria ver o que se faria, porque as vezes só tem ela para cuidar daquela pessoa (Gestor, 38 anos, Fortaleza).

A saúde do cuidador e seu acesso ao serviço também é um fator a ser considerado pela APS. O cuidador familiar costuma ser usuário da mesma equipe de saúde do idoso, embora esse cuidador não pareça ser abordado na visita domiciliar como usuário da unidade, com suas próprias demandas, e sim como informante das condições de saúde do idoso dependente.

Os cuidados domiciliares, técnicos ou leigos, também acontecem como forma de proteção social, no monitoramento dos fatores de riscos e desenvolvimento de ações de prevenção de doenças ou agravos. Em alguns casos, o próprio cuidador também é um idoso. Por conseguinte, o acompanhamento destes idosos e cuidadores familiares pelas equipes poderia funcionar como uma forma de proteção e prevenção, tendo em vista que o trabalho pode gerar fragilidade na saúde física e mental. O cuidado do idoso dependente afeta aspectos emocionais e afetivos da vida do cuidador, além de aspectos sociais, já que implica em um redimensionamento de sua vida, com restrições em diversos campos, como lazer e vida pessoal ${ }^{34}$.

Pereira e Soares ${ }^{35}$ concluíram que a literatura sobre esse tema é escassa nacional e internacionalmente, sendo fundamental discutir a qualidade de vida desses cuidadores e as políticas e programas voltados para esse público. $\mathrm{O}$ acesso à APS figurou como um dos fatores identificados no estudo que influencia diretamente a qualidade de vida do cuidador familiar, no tocante à sobrecarga de trabalho, à dificuldade de ir e vir, ao receio em deixar o idoso sozinho e às repetidas jornadas à unidade de saúde para agendar con- 
sultas. Porém, a inexistência de políticas voltadas às famílias e aos cuidadores dos idosos e, consequentemente, seu acesso ao serviço de saúde, fica restrito aos fluxos específicos das unidades ${ }^{36}$.

A possibilidade de a APS estar perto das famílias e comunidade propiciaria a resolutividade da maioria dos problemas de saúde da população e o impacto na redução custo-efetividade. $\mathrm{O}$ cuidado domiciliar, quando adequadamente organizado, pode ampliar a autonomia dos idosos e de seus cuidadores, reduzir as hospitalizações e as complicações decorrentes, como a mortalidade e os custos financeiros e psicológicos. O cuidado domiciliar, deste modo, é uma abordagem custo-efetiva e pode, ainda, ajudar a garantir a integralidade ${ }^{30}$.

O cuidado domiciliar é uma prática de humanização da assistência, determinante para a formação e condição do trabalho vivo, uma tecnologia sofisticada e complexa de cuidado em saúde. Entretanto, os casos de idosos dependentes e as relações estabelecidas no cotidiano do domicílio entre cuidadores e equipe revelam a necessidade de redes sociais protetivas e de apoio no âmbito macro e micropolítico. Essas concepções reafirmam o papel do cuidado domiciliar para a atenção ao idoso dependente e cuidador familiar a partir de duas dimensões: 1) integralidade no cuidado e 2) proteção social no cuidado. Assim, é importante reafirmar a necessidade de uma análise permanente da atenção à saúde do cuidador e do idoso dependente e no acesso ao cuidado domiciliar oferecido ${ }^{30}$.

As discussões acerca do acesso e do cuidado domiciliar de idosos dependentes e seus cuida- dores à APS suscita questões diversas e complexas, pois o cuidado a este grupo desafia muitas das estruturas já estabelecidas no cotidiano da rede de serviços de saúde. Assim, este artigo não se propõe a findar tal discussão, mas fomentá-la, trazendo à luz da literatura questões ainda pouco discutidas, apesar do crescimento populacional de idosos no Brasil e no mundo.

\section{Considerações Finais}

Este estudo evidencia fragilidades da APS no cuidado ao idoso dependente e aos seus cuidadores na maioria dos municípios investigados. Entretanto, configura-se como um modelo assistencial imprescindível para a integralidade da atenção à saúde, com potencialidade de atender a maioria das necessidades vinculadas a essa população.

Problemas no acesso, na atenção domiciliar, na rede de atenção à saúde e no trabalho interprofissional na APS foram observados no estudo. Além do mais, as equipes ofertam práticas sob a lógica do modelo biomédico e centradas no profissional médico, embora tenham sido identificadas ações de promoção de saúde e prevenção de doenças, principalmente no âmbito do domicílio e realizadas pelos agentes comunitários de saúde.

Há necessidade de qualificar a APS e ampliar o escopo de práticas, incorporando núcleos de saberes que não estão tradicionalmente inseridos nas equipes. Além do mais, é fundamental o fortalecimento do papel do Estado e a criação de políticas públicas específicas para os idosos dependentes e seus cuidadores. 


\section{Colaboradores}

RF Ceccon, CCSA Matos, CAS Garcia Júnior trabalharam na concepção, análise, interpretação dos dados, redação inicial e final; LJES Vieira participou da interpretação dos dados, revisão crítica e redação final; MDHA Pascoal e KG Soares colaborou na análise, compilação e interpretação inicial dos dados.

\section{Referências}

1. Minayo MCS, Firmo JOA. Longevidade: bônus ou ônus? Cien Saude Colet 2019; 24(1):4.

2. Klompstra L, Ekdahl AW, Krevers B, Milberg A, Eckerblad J. Factors related to health-related quality of life in older people with multimorbidity and high health care consumption over a two-year period. BMC Geriatr 2019; 19(1):1471-2318.

3. Brasil. Constituição da República Federativa do Brasil. Brasília: Centro Gráfico do Senado Federal; 1988.

4. Batista AS, Jaccoud LB, Aquino L, El-Moor PD. Envelhecimento e dependência: desafios para a organização da proteção social. Brasília: MPS, SSP; 2008. (Coleção Previdência Social).

5. Starfield B. Atenção Primária: equilíbrio entre necessidades de saúde, serviços e tecnologia. Brasília: Unesco, Ministério da Saúde; 2002.

6. Brandão JRM. A atenção primária à saúde no Canadá: realidade e desafios atuais. Cad Saude Publica 2019; 35(J):e00178217.

7. Placideli N, Castanheira ERL, Dias A, Silva PA, Carrapato JLF, Sanine PR, Machado DF, Mendonça CS, Zarili TFT, Nunes LO, Monti JFC, Hartz ZMA, Nemes MIB. Avaliação da atenção integral ao idoso em serviços de atenção primária. Rev Saude Publica 2020; 54(6):1-14.

8. Minayo MCS. O desafio do conhecimento. Pesquisa qualitativa em saúde. São Paulo: Hucitec; 2010.

9. Minayo MCS. Estudo situacional dos idosos dependentes que residem com suas famílias visando subsidiar uma política de atenção e de apoio aos cuidadores. Rio de Janeiro: Fiocruz; 2019. (Projeto de Pesquisa).

10. Minayo MCS. O imperativo de cuidar da pessoa idosa dependente. Cien Saude Colet 2019; 24(1):247-252.

11. Instituto Brasileiro de Geografia e Estatística (IBGE). Características gerais dos moradores 2012-2016. Rio de Janeiro: IBGE; 2017.

12. Brasil. Portaria no 2.528 , de 19 de outubro de 2006 . Aprova a Política Nacional de Saúde do Idoso. Diário Oficial da União 2006; 20 out.

13. Campos GWS. Saúde pública e saúde coletiva: campo e núcleo de saberes e práticas. Cien Saude Colet 2000; 5(2):219-230.

14. Oliveira HM, Moretti-Pires RO, Parente RCP. As relações de poder em equipe multiprofissional de Saúde da Família segundo um modelo teórico arendtiano. Interface (Botucatu) 2011; 15(37):539-550.

15. Santos AM, Giovanella L, Mendonça MHM, Andrade CLT, Martins MIC, Cunha MS. Práticas assistenciais das Equipes de Saúde da Família em quatro grandes centros urbanos. Cien Saude Colet 2012; 17(10):26872702.

16. Moraes GVO, Giacomin K, Santos WJ, Firmo JOA. A percepção dos idosos sobre o saber biomédico no cuidado à velhice e às "coisas da idade". Physis 2016; 26(1):309-329.

17. Makary MA, Daniel M. Medical error: the third leading cause of death in the US. BMJ 2016; 353:i2139.

18. Merhy EE. Saúde: a cartografia do trabalho vivo. São Paulo: Hucitec; 2002.

19. Rose N. Beyond medicalisation. Lancet 2007; $369(\mathrm{XX}): 700-701$. 
20. Marin MJS, Cecílio LCO. Necessidades de saúde de idosos de uma Unidade de Saúde da Família. Rev Bras Geriatr Gerontol 2009; 12(1):63-76.

21. Seixas CT, Baduy RS, Cruz KT, Bortoletto MSS, Junior HS, Merhy EE. O vínculo como potência para a produção do cuidado em Saúde: o que usuários-guia nos ensinam. Interface (Botucatu) 2019; 23:e170627.

22. Magalhães KA, Giacomin KC, Santos WJ, Firmo JOA. A visita domiciliária do agente comunitário de saúde a famílias com idosos frágeis. Cien Saude Colet 2015; 20(12):3787-3796.

23. Cunha MS, Sá MC. A visita domiciliar na Estratégia de Saúde da Família: os desafios de se mover no território. Interface (Botucatu) 2013; 17(44):61-73.

24. Melo EA, Mendonça MHM, Oliveira JR, Andrade GCL. Mudanças na Política Nacional de Atenção Básica: entre retrocessos e desafios. Saúde Debate 2018; 42(n. esp. 1):38-51.

25. Cunha GT, Campos GWS. Apoio Matricial e Atenção Primária em Saúde. Saude Soc 2011; 20(4):961-970.

26. Brasil. Ministério da Saúde (MS). Portaria no 2.979, de 12 de novembro de 2019. Diário Oficial da União 2019; 13 nov.

27. Schenker M, Costa DH. Avanços e desafios da atenção à saúde da população idosa com doenças crônicas na Atenção Primária à Saúde. Cien Saude Colet 2019; 24(4):1369-1380.

28. Brasil. Ministério da Saúde (MS). Portaria no 2.436, de 21 de setembro de 2017. Diário Oficial União 2017; 22 set.

29. Brasil. Ministério da Saúde (MS). Atenção domiciliar às pessoas idosas. In: Brasil. Ministério da Saúde (MS). Envelhecimento e saúde da pessoa idosa. Brasília: MS; 2006. (Caderno de Atenção Básica no 19). p. 125127.

30. Brasil. Ministério da Saúde (MS). Caderno de Atenção Domiciliar. Brasília: MS; 2012. v. 2.
31. Martins AB, D’Avila OP, Hilgert JB, Hugo FN. Atenção Primária à Saúde voltada às necessidades dos idosos: da teoria à prática. Cien Saude Colet 2014, 19(8):34033416.

32. Mazza MMPR, Lefèvre F. Cuidar em família: análise da representação social da relação do cuidador familiar com o idoso. Rev Bras Cresc Desenvolv Hum 2005; 15(1):1-10.

33. Besse M, Cecílio LCO, Lemos ND. A equipe multiprofissional em gerontologia e a produção do cuidado: um estudo de caso. Revista Kairós Gerontologia 2014; 17(2):205-222.

34. Anjos KF, Boery RNSO, Pereira R. Qualidade de vida de cuidadores familiares de idosos dependentes no domicílio. Texto Contexto Enferm 2014; 23(3):600608.

35. Pereira LSM, Soares SM. Fatores que influenciam a qualidade de vida do cuidador familiar do idoso com demência. Cien Saude Colet 2015; 20(12):3839-3851.

36. Fernandes MTO, Soares SM. O desenvolvimento de políticas públicas de atenção ao idoso no Brasil. Rev. Esc. Enferm. USP 2012; 46(6):1494-1502.

Artigo apresentado em 22/07/2020

Aprovado em 11/08/2020

Versão final apresentada em 13/08/2020

Editores chefes: Maria Cecília de Souza Minayo, Romeu Gomes, Antônio Augusto Moura da Silva. Editora Associada da área de Saúde do Idoso: Joselia Oliveira Araújo Firmo 